\title{
Concepts About the Causes of Development: Travel, Visual Experience, and the Development of Dynamic Spatial Orientation
}

\author{
John J. Rieser \\ Department of Psychology and Human Development \\ Vanderbilt University
}

\begin{abstract}
"Travel broadens the mind" is a good concept for developmental psychology. Travel is a metaphor for human experience as we explore the paths that life provides and theorize about the roles the paths play in development. The idea is one that I first learned about over dinner conversations as a child. The idea comes up over and over in literature and shaped many of us, reading Heyerdal's (1948) The Kon Tiki Expedition and Steinbeck's (1962) Travels With Charley in middle school, Dana's (1840) Two Years Before the Mast and Darwin's (1909) Voyage of the Beagle in high school, Henry James's (1885) A Little Tour of France and Orwell's (1933) Down and Out in Paris and London in college.
\end{abstract}

For Campos et al. (this issue), travel is not a metaphor, but instead a summary label for the host of specific experiences that typically occur together with indeendent locomotion. Their article is about what we accept as explanation in psy chological development. They spell out some of the specific experiences that are associated with locomotion and point out processes by which these experiences might be linked together in a causal chain relating those specific experiences to development of depth perception, dynamic spatial orientation, emotion, and social relations.

What makes this article important is the concept of psychological explanation that it provides. The rest of this article consists of two sections. The first is focused on

Requests for reprints should be sent to John J. Rieser, Department of Psychology and Human Development, Vanderbilt University, 2200 West End Avenue, Box 512 Peabody, Nashville, TN 37203. E-mail: rieserjj@ctrvax.vanderbilt.edu 
earlier theories and explanations of development. Campos et al.'s (this issue) concepts of causes and explanation build on earlier works, and I relate them to three sets of ideas about the causes of development that emerged about 25 years ago. The second section is an illustration of how Campos et al.'s concepts of causality apply to my own work linking the visual experiences available during locomotion to the development of dynamic spatial orientation when walking without vision.

\section{EXPLANATION AND CAUSALITY IN THEORIES OF DEVELOPMENT}

Wohlwill (1973) published an article entitled "The Concept of Experience: S or R?" A main theme was that experience was too often thought of as something that happened to children. Wohlwill argued that children actively shape their own experiences, for example, by selecting them, by selectively attending to them, and by shaping the specifics of the experience through the details of their own actions. Wohlwill created four metaphors to help developmentalists think more clearly about the ways children can participate in life. These are a hospital bed (children lie in bed and watch, listen, and learn), an amusement park (children pick and choose among the rides they encounter as they walk through life, but once they pick a particular experience they do not influence its outcome), a swim meet (children decide to enter the swim meet and their behavior influences the outcome of who wins, but it does not influence the details of the process), and a tennis match (children decide to enter the tennis match and in addition the details of how they play influence their opponent's play, which determines the final winner). Wohlwill emphasized the ways that children reciprocally shape and are shaped by their life experiences.

Despite his emphasis on the reciprocal relation of child and environment, Wohlwill (1973) did not emphasize the ways those reciprocal relations can change with age. This was a main point of Sameroff's (1975) article entitled "Early Influences on Development: Fact or Fancy?". It focused on his review of research about the persistence of perinatal complications and abuse. He argued that the effects of experiences such as perinatal complications and abuse are better thought of as reciprocal transactions, not as a main effect of the environment and not as a simple time-limited interaction of children with their environments.

Whereas Sameroff did not tease apart different categories of effect that experience could have on behavioral development, this was a main point of Gottlieb's (1976) article entitled "Conceptions of Prenatal Development: Behavioral Embryology." It focused on a review of research about the development of species-typical behaviors, especially the development by different birds of their species-typical song. One of his main points was that genetics acts in concert with experience to codetermine behavioral development. Gottlieb argued that experience acts to maintain a behavior that occurs without experience but will become 
diminished without it, to facilitate a behavior either by speeding its acquisition or causing more of it to develop, and to induce a behavior or quality of behavior either by causing more of it to develop or by causing a particular form of the behavior.

Whereas Gottlieb (1976) only sketched different specific causal processes linking experience with learning and psychological development, elaborating the details of these linking processes is a main point of Campos et al. Campos et al.'s target article is organized around their cumulative studies linking the onset and practice of locomotion with the acquisition of some of the basic mental skills achieved by infants, including depth perception as assessed by what happens on the visual cliff, spatial orientation as assessed by where infants search for expected hidden events after they are moved to a new observation point, and emotional regulation and social referencing as assessed by the degree to which infants look toward adults in situations where they are faced with a novel event that might be dangerous. One of their main points is that the onset of locomotion, a ubiquitous developmental accomplishment, causes or is correlated with a family of experiences, and each member of the family contributes to the development of some behaviors but not to others. Even though a number of psychological phenomena are related to a single pacer or organizer, each outcome can be dissociated from the other, so the outcomes need not be correlated despite a common influence.

According to the theory the acts of locomotion (e.g., balancing while being carried, scooting, crawling, and walking) can themselves be causes of development, and in addition they can set the stage for or increase the probability of other experiences. These other experiences, in turn, link together, creating a chain of causal processes explaining what was learned. One can think of it in terms of "proximal" and "distal" causality, wherein the proximal cause links directly to psychological processes causing developmental change, whereas the distal cause enables the proximal cause to take place. Consider the following syllogism about a possible explanation of the causes linking locomotion with the development of spatial orientation:

1. If genetic variability causes differences that result in some infants walking at earlier ages than other infants.

2. If experience walking provides chances to learn and to attend to the correlation of optical-environmental flow with locomotion, and this learning, in turn, accounts for dynamic spatial orientation while walking without vision.

3. Therefore it follows that genetic differences (those that directly influence that age of walking) cause individual differences in the development of spatial orientation.

Yes, it follows, but only indirectly, because according to the theory it is the chance to experience and learn about the correlation of optical-environmental flow with locomotion that directly accounts for spatial orientation. 
According to Campos et al., locomotor experience is neither necessary nor sufficient for producing psychological change. They give four reasons for why locomotion, or any other category of experience, can be important for development, but not a necessary or sufficient cause:

1. Principle of partial accomplishment: Experience acts to facilitate and improve the development of skills that emerge without it.

2. Principle of precocious exposure: The specific experiences that serve as the proximal causes of development can occur without locomotion, and thus infants who are too young to locomote (or cannot locomote) can experience the same specific experiences and develop skills.

3. Principle of equipotentiality: Particular psychological outcomes can result from different processes than those linked to locomotion.

4. Principle of maintenance by experience: Experience might not be needed to induce or facilitate a psychological skill, but instead it might be needed to update the skill and to prevent its eventual loss from disuse.

\section{DYNAMIC SPATIAL ORIENTATION WHEN WALKING WITHOUT VISION: WHAT DEVELOPS?}

When locomoting without vision, adults and young children are able to keep up-to-date on the dynamically changing distances and directions relative to objects in their remembered surroundings. Dynamic spatial orientation refers to awareness of the network of changing self to object distances and directions that occur whenever one locomotes. The development of dynamic spatial orientation involves learning about the qualitative differences involved in the geometry of rotational and of transnational locomotion, and it involves learning the quantitative relations linking distances turned and walked to the corresponding changes in perspective. Consider the learning of these qualitative and quantitative relations each in turn (Rieser, 1999).

In terms of qualities, skillful, dynamic spatial orientation involves knowing about the geometry of self-movements-knowing, for example, that simple locomotor rotations result in rotations in the self-to-object directions and no change in the distances, and that simple locomotor translations result in changes in self-to-object distances and directions that depend on the particular object's distance from the person and direction relative to the direction of locomotion. In terms of quantities, skillful dynamic orientation depends on fine-tuning the calibration of locomotion rates relative to rates change in orientation-how much change in self-to-object direction is caused by a given motoric rate of turning, and how much change in self-to-object distances and directions is caused by a given motoric rate of forward walking? 
Campos et al. review their studies linking independent locomotion during the first years of life to the mastery of a handful of important developmental skills. They show there is a moderate correlation linking measures of age of independent locomotion with the handful of skills. In addition, they show that experimental manipulation of the age of independent locomotion is functionally linked to the age of acquiring the skills (they demonstrated earlier skill acquisition of infants who used "walkers" to get around before they could walk than of infants who did not use walkers). Empirically, Campos et al. are aware of exceptions to the idea that locomotion plays a critical role in development, exceptions, for example, of children who lack limbs or are unable to walk. Theoretically, they cannot argue that locomotion is either a necessary cause or a sufficient cause of the development of these skills. What they do instead is better-they give examples of causal chains (Quine \& Ullian, 1978) linking specific opportunities afforded by locomotion with processes that cause learning and skill development. Children who do not locomote can have some of the same experiences as the experiences typically afforded by locomotion, or experiences that can substitute for them. Their claim is not that locomotion per se is necessary, but instead that locomotion is associated with more specific experiences, and these specific experiences can participate in the processes of learning and change.

The dynamic optical flow that occurs during locomotion is an example of a type of specific experience that is occasioned by locomotion that might play a role in the development of dynamic spatial orientation. I turn now to research probing the relation of visual experience and spatial development.

\section{Empirically, Visual Experience Plays a Role in the Development of Skillful Dynamic Spatial Orientation}

Visual experience plays a role in the development of dynamic spatial orientation, though not in the case of every individual. As a group, children and adults who were born without vision are typically deficient compared to those who lose vision after childhood (Millar, 1996; Rieser, Guth, \& Hill, 1986; Thinus-Blanc \& Gaunet, 1997). However, it is also clear that visual experience is not necessary, even for the development of the highest levels of skill. The results of a recent unpublished Vanderbilt study of path integration by 40 adults who were congenitally blind and 25 who became blind after early childhood provide an example of this. During each of the repeated trials of the task, the blind participants followed a sighted guide on routes that varied from three to seven turns and then judged the direction back to the starting position of the route. There was a large range of variability, with the most skilled performer averaging 8 degrees of error across the repeated trials and the least skilled averaging 111 degrees of error. Consider three facts about the findings and their corresponding implications. First, the participants who became blind relatively 
late in life (after 8 years of age in our sample) were significantly more accurate than those who were born without vision. This indicates that visual experiences (i.e., learning opportunities that can be correlated with being able to see during childhood) play a role in the development of path integration. Second, visual experience is not necessary for people to achieve the highest levels of performance on path integration, because two of the top five scorers were congenitally blind persons. Third, the worst five scorers were all congenitally blind and their errors were all greater than the 90 degrees of error a person would expect by someone who simply guessed, whereas the greatest error earned by the late-blinded participants was 45 degrees.

\section{A Perceptual-Motor Learning Theory}

Walking with vision provides optical flow input that specifies the qualitative differences in spatial orientation between rotations and translations. The idea is that when walking with access to vision, people notice how the biomechanical features of their walking correlate with the optical flow. Then when walking without vision, their spatial orientation is mediated by this learning. So, for example, when rotating by turning in place, they readily see that the self-to-objects distances all change at the same rate across the relatively broad field of view of vision. In addition, when translating by walking forward, they see that the distances and directions to nearby objects that are off their paths undergo faster rates of change than those of faraway objects. Furthermore, when walking with access to vision, people notice how the biomechanical rate of their walking correlates with the optical flow rate specifying the changing self-to-object distances and directions, and this learned correlation, in turn, determines their dynamic spatial orientation (Rieser, Pick, Ashmead, \& Garing, 1995).

Empirical evidence indicates that visual experience is not necessary for the development of dynamic spatial orientation, because some persons who are congenitally blind develop superb dynamic spatial orientation skills. I surmise that there are nonvisual opportunities to learn the qualitative and quantitative relations of good orientation, because the superbly skilled congenitally blind participants understood the geometry of rotations and translations and their locomotion was accurately calibrated relative to the routes they walked. The evidence shows the probability of a high level of learning is greater with visual experience. Visual experience might, however, be a sufficient basis for learning the qualitative relations, as all of the late-blinded participants responded with below chance levels of error. Nor is visual experience necessary for the development of fine-tuning the calibration of locomotor actions, again, because some of the congenitally blind participants scored with the highest levels of accuracy, nor is it sufficient for the highest levels of fine-tuning, because there was a broad range of error among those who were blinded after childhood. 


\section{How Exactly Might Visual Experience Matter?}

The perceptual-motor learning theory of the development of dynamic spatial orientation implies that broad visual fields are important and that information about self-to-object distances is important. The reasons for both hinge on the geometry of translations. When walking along a straight line, the rates at which self-to-object directions and distances change depend on the particular object's direction relative to the direction of walking (e.g., objects that are straight ahead do not change in their direction, whereas objects that are not straight ahead do change). Vision has a broad field of perception, processes multiple features in parallel, and seems better suited than other types of environmental input at specifying how these rates of change vary for things ranging from the central to peripheral field. In addition, when walking a straight line, the rates at which self-to-object distances and directions change depend on the particular object's distance (e.g., nearby objects change their bearing relative to a moving observer more quickly than faraway objects). Vision has a deep field of view and again seems better suited than other senses for detecting the distances of multiple objects in parallel.

\section{People With Congenitally Small Visual Fields, Like People Who Are Congenitally Blind, Tend to Show Poor Spatial Learning}

Rieser, Hill, Talor, Bradfield, and Rosen (1992) conducted a naturally occurring groups experiment in the field to test this theory. The idea was that if a broad and differentiated perceptual field is critical to the development of dynamic spatial orientation, then people who are born with small visual fields should be at a disadvantage relative to those born with normal vision and those born with other visual abnormalities. All participants in the study were asked to judge the straight-line directions relating objects in a four-square-block region of a very familiar neighborhood, one where they could travel skillfully among all the landmarks in the region. The idea was that people who are skillful at integrating the distances and directions across the circuitous paths typically walked in neighborhoods would know these straight-line directions, whereas people who were not skillful at path integration would not know these directions. The participants included adults who were blind (some were born without vision, others became blind after about 8 years of age), adults with small visual fields (some born with small fields, others acquiring them after about 8 years of age), and adults with normal visual fields but very poor acuity (again, some born with poor acuity, others acquiring it after 8 years of age).

The results showed very poor performance by the group of congenitally blind participants and the congenitally small visual field participants. The groups of participants with late-onset blindness and late-onset small fields both performed rela- 
tively well. Finally, the groups with early as well as late-onset poor acuity performed relatively well. The pattern of findings has a specific fit with the perceptual-motor learning theory.

\section{A FINALE IN TWO QUESTIONS}

The target article by Campos et al. provides good examples of the types of causal chains linking the experiences of children's daily lives to their mental accomplishments that developmental psychology needs. I close with two questions about the scientific adequacy of the theory. The first question is this: If a theory posits that something is neither a necessary nor a sufficient cause for something else, then what is it? Campos et al.'s answer to this question for the "something" of locomotion and the "something else" of mental development is that the specific experiences that are typically afforded by locomotion link directly with psychological development. The second question is this: If a theory posits that something is neither a necessary nor sufficient cause of something else, then how could it in principle be falsified? Campos et al.'s answer to this question is that the details matter. I think they have made great progress in investigating the detailed connections they theorize link locomotion with psychological development.

\section{REFERENCES}

Gottlieb, G. (1976). Conceptions of prenatal development: Behavioral embryology. Psychological Review, 83, 215-234.

Millar, S. (1994). Understanding and representing space: Theory and evidence from studies with blind and sighted children. New York: Oxford University Press.

Quine, W. V., \& Ullian, J. S. (1978). The web of belief (2nd ed.). New York: McGraw-Hill.

Rieser, J. J. (1999). Dynamic spatial orientation and the coupling of representation and action. In R. Golledge (Ed.), Cognitive mapping and spatial behavior (pp. 168-190). Baltimore: Johns Hopkins Press.

Rieser, J. J., Guth, D. A., \& Hill, E. W. (1986). Sensitivity to perspective structure while walking without vision. Perception, 15, 173-188.

Rieser, J. J., Hill, E. W., Talor, C. R., Bradfield, A., \& Rosen, S. (1992). Visual experience, visual field size, and the development of nonvisual sensitivity to the spatial structure of outdoor neighborhoods explored by walking. Journal of Experimental Psychology: General, 121, 210-222.

Rieser, J. J., Pick, H. L., Jr., Ashmead, D. A., \& Garing, A. E. (1995). The calibration of human locomotion and models of perceptual-motor organization. Journal of Experimental Psychology: Human Perception and Performance, 21, 480-497.

Sameroff, A. J. (1975). Early influences on development: Fact or fancy? Merrill-Palmer Quarterly, 21, 267-294.

Thinus-Blanc, C., \& Gaunet, F. (1997). Representations of space in blind people: Vision as a spatial sense? Psychological Bulletin, 121, 20-42.

Wohlwill, J. F. (1973). The concept of experience: S or R? Human Development, 16, 90-107. 